\title{
EDITORIAL
}

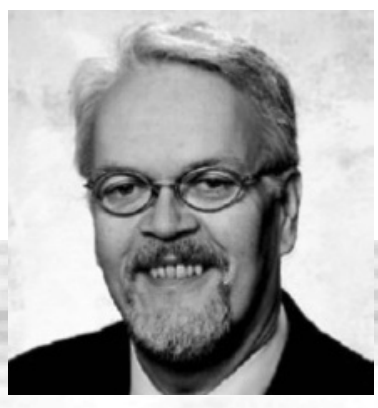

\section{Hermann Engesser}

Chefredakteur

\section{Die sich verändernde Rolle der Informatik}

Die Informatik und der damit verbundene Spielemarkt entwickeln sich rasant. Das von Nintendo und dem GoogleUnternehmen Niantic für iOS- und Android-Smartphones entwickelte mobile Spiel Pokémon GO erobert mit einer enormen Geschwindigkeit den Weltmarkt. Pokémon GO ist ein Augmented-Reality-Spiel, bei dem die reale Welt als Grundlage genommen und durch zusätzliche Daten angereichert wird. Im Fall von Pokémon GO nutzt Niantic die Karten von Google, um den Spielern eine virtuelle Welt anzuzeigen, welche der realen Welt in ihren Grundzügen (Topografie, Straßen, Wege) gleicht.

Jochen Krüger, Frederik Möllers und Stephanie Vogelgesang stellen in ihrem Beitrag Pokémon GO - Technische Innovation und Strafrecht im Konflikt zunächst die technischen Grundlagen dieses Spiels und seine Funktionsweise dar. Anschließend beschreiben sie verschiedene Gefährdungslagen für die Spieler und die Umgebung und würdigen diese in strafrechtlicher Hinsicht.

Im Interview diskutieren Manfred Broy und Christian Prehofer über die Digitalisierung und die sich verändernde Rolle der Informatik in Anwendung und Forschung. Um die Rolle der Informatik und die Herausforderungen durch die Digitalisierung genauer zu verstehen, erläutern sie im Gespräch die typischen Phasen der Digitalisierung, von der Digitalisierung bestehender Prozesse bis zur Erarbeitung neuer digitaler Geschäftsmodelle und Ökosysteme. Informatik ist eine notwendige Voraussetzung für die aktive Ausgestaltung von neuen digitalen Geschäftsmodellen. Software und Daten wandeln sich damit von einem reinen "Enabler" zu einem strategischen, geschäftsbestimmenden Asset. Aus dieser Perspektive entstehen neuen Herausforderungen für die Informatik sowohl in Forschung und Lehre, als auch in der Praxis.

Der Begriff "Cyberspace" tauchte erstmals in den 1980er-Jahren in der ScienceFiction-Literatur auf. Gemeint war damals ein von der echten Welt verschiedener, virtueller Raum, in dem andere Regeln galten.

Mittlerweile bemerken wir, dass es diese andere Welt gar nicht gibt, sondern dass

\section{"On the internet, nobody knows you're a dog." \\ Peter Steiner} das Digitale ein Teil unserer gewöhnlichen Welt ist. Niemand ist mehr "online" oder "offline", weil auch die mobilen Geräte per default verbunden und ohne Netzverbindung teilweise völlig nutzlos sind. Bei „digitalen Aktivitäten“ geht es immer auch um reale Aktionen und reale Konsequenzen. Reiner Rehak stellt in seinem Artikel Der irreführende „Cyberspace“ - wenn Begriffe Verantwortlichkeiten verschleiern die Herausforderungen zur Diskussion, die erforderlich sind, um die Digitalisierung der Gesellschaft vernünftig zu gestalten.

Heinz-Jürgen Burkhardt und Rainer Prinoth betonen in ihrer Notiz Von Sachertorte und anderen Dingen ingenieurwissenschaftliche Grundsätze zu einer Verbesserung der IT-Sicherheit. Anhand von Beispielen empfehlen sie, dass für Arbeitskontexte jeweils eigene, auf diese zugeschnittene Ausführungsumgebungen bereitgestellt werden. Die heutige IT stellt derartige Arbeitsumgebungen, in der Arbeitskontexte strikt voneinander getrennt sind, allerdings nicht zur Verfügung. Sie ist darauf ausgerichtet, unaufwendig alles mit allem und dies jederzeit verknüpfen zu können. Dadurch wird sie angreifbar, manipulierbar, für ihre Entwickler kaum beherrschbar und für ihre Nutzer weder durchschau- noch kontrollierbar. Die Autoren fordern daher zumindest in sicherheitskritischen Arbeitskontexten eine Abkehr von dem in der heutigen IT praktizierten Bottom-up-Ansatz hin zu einem auf Arbeitskontexte zentrierten Top-down-Ansatz, in dem Ausführungsumgebungen dynamisch auf Arbeitskontexte konfigurierbar sind. 


\section{\{ EDITORIAL}

Timm Grams beschreibt in seiner historischen Notiz So rechnen Zuse-Computer - und die heutigen auch ein Addierermodell des mechanischen Computers Z1, das die grundlegende Arbeitsweise des rein mechanisch arbeitenden Zuse-Rechners Z1 sichtbar macht. Dabei steht die Didaktik im Vordergrund, denn es ist insbesondere die Mechanik des ersten Rechners, die Mathematik und Informatik begreifbar macht. Das Z1-Addierermodell erlaubt die Demonstration der zentralen Funktionsprinzipien eines jeden Computers.

Im aktuellen Schlagwort OCR für alte Drucke beschreibt Uwe Springmann einen Weg zu einer digitalen WeltBibliothek, in der u. a. Volltextsuche durchgehend möglich wird. Noch ist der größte Teil des schriftlichen kulturellen Erbes in gedruckten Büchern gespeichert, die seit der Erfindung des Buchdrucks mit beweglichen Metallettern vorliegen. In mehreren Digitalisierungsprojekten wird daran gearbeitet, digitale Seitenbilder dieser Bücher durch Anwendung der Buchscantechnologie zu erzeugen, um praktisch alle jemals hergestellten Buchtitel in wenigen Jahren vollständig zu digitalisieren und für die Allgemeinheit im Internet verfügbar zu machen. Beim Scannen nimmt man jedoch in Kauf, dass die Bildseiten nicht maschinell durchsucht werden können. Von einer echten digitalen Universalbibliothek kann man erst sprechen, wenn die Texte auch in maschinenverarbeitbarer Form vorliegen, damit Fundstellen, Querverweise, Abhängigkeiten und die ganze Breite des intellektuellen Diskurses auf Knopfdruck zur Verfügung stehen. Die Anfang des 20. Jahrhunderts erfundene Technologie der optischen Zeichenerkennung (Optical Character Recognition, $O C R$ ) ist hier von zentraler Bedeutung. Uwe Springmann erläutert den in den letzten Jahren erzielten Durchbruch auf dem Gebiet der OCR, alte Drucke auf der Basis von rekurrenten neuronalen Netzen zu erschließen.

Auch bei den anderen Beiträgen viel Lesevergnügen.

\section{Hermann Engesser}

